\title{
An intrinsic correlation between GRB optical/UV afterglow brightness and decay rate
}

\author{
S. R. Oates ${ }^{* 1}$, M. J. Page ${ }^{1}$, M. De Pasquale ${ }^{2}$ and P. Schady ${ }^{3}$ \\ ${ }^{1}$ Mullard Space Science Laboratory, University College London, Holmbury St. Mary, Dorking \\ Surrey, RH5 6NT, UK \\ ${ }^{2}$ University of Nevada, Las Vegas, Department of Physics \& Astronomy, Box 454002, 4505 \\ Maryland Parkway, Lax Vegas, NV 89154-4002 \\ ${ }^{3}$ Max-Planck Institut für Extraterrestrische Physik, Giessenbachstraße 1, 85748 Garching, \\ Germany \\ E-mail: sro@mssl.ucl.ac.uk
}

\begin{abstract}
We examine a sample of 47 Swift/UVOT long Gamma-ray Burst light curves. Using the restframe light curves we find a correlation between the logarithmic luminosity determined at 200s, $\log \mathrm{L}_{200 \mathrm{~s}}$, and average decay rate measured from 200s onwards, $\alpha_{>200 \mathrm{~s}}$. The Spearman rank coefficient for the correlation is -0.54 at a probability of $99.99 \%(3.9 \sigma)$. We find the $\log \mathrm{L}_{200 \mathrm{~s}}-\alpha>200 \mathrm{~s}$ correlation to be an intrinsic property of long GRBs and discuss three potential causes. Of these three scenarios, we are able to exclude the correlation as resulting naturally from the standard afterglow model. However, we cannot presently exclude or distinguish between the other two possibilities. The first scenario is that there is some property of the central engine, outflow or external medium that affects the rate of energy release in such a way that the bright afterglows release their energy more quickly than the fainter afterglows. Alternatively the correlation may be produced by variation of the observers viewing angle, with observers at large viewing angles observing fainter and slower light curves.
\end{abstract}

Gamma-Ray Bursts 2012 Conference-GRB2012,

May 07-11, 2012

Munich, Germany

\footnotetext{
* Speaker.
} 


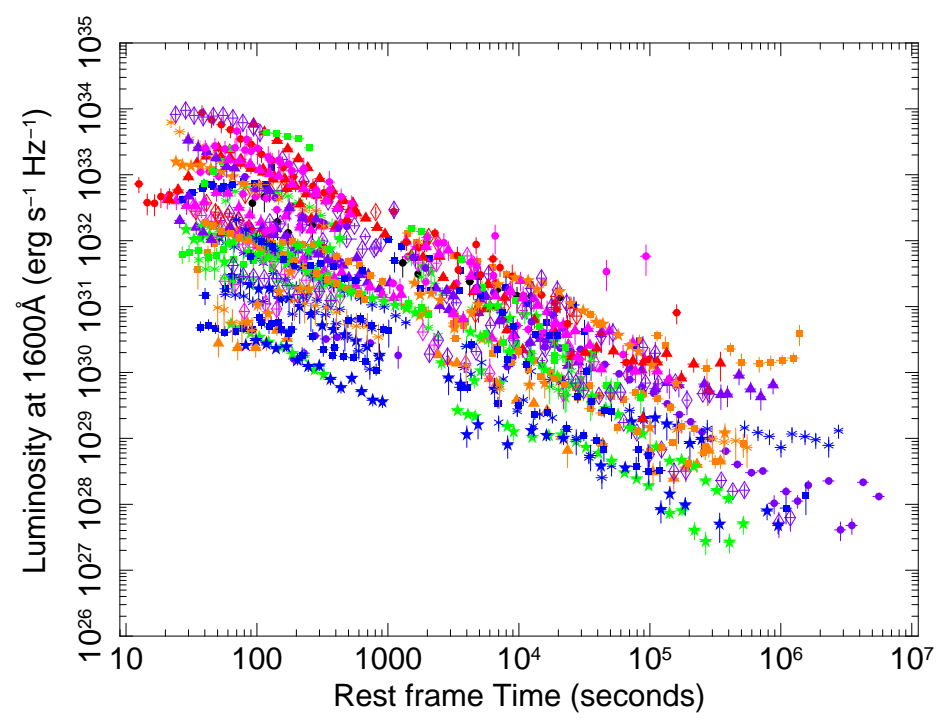

Figure 1: Luminosity light curves of 56 GRBs at $1600 \AA$. For clarity the $3 \sigma$ upper limits are not included.

\section{Introduction}

With over 600 Gamma-ray Bursts (GRBs) detected by Swift [四, we now have a sample of IR/optical/UV light curves large enough to begin probing the characteristics of the afterglow at these frequencies and from which we can start to understand, from the optical perspective, the afterglow emission mechanisms and the structure of the emitting outflow. In 2009, we performed a statistical analysis on a small sample of 26 optical GRB light curves [⿰]月 a correlation between the observed $v$-band magnitude at 400s and the average UVOT light curve decay rate determined from 500s. However, to have implications on our understanding of GRBs this correlation must be observed in the rest frame. Using this sample we tested for an equivalent rest frame correlation, but this could not be confirmed or excluded, due to the small sample size. Here we use a larger sample of 47 high quality long GRB UVOT light curves to re-examine if there is a correlation between optical/UV afterglow intrinsic brightness and light curve decay rate.

\section{Sample Selection}

Using the second UVOT GRB catalogue [3], we have collated a sample of 69 light curves, which were selected by the criteria given in [2]: the optical/UV light curves must have a peak UVOT $v$-band magnitude of $\leq 17.89$ (equivalent to $\geq 1$ count $\mathrm{s}^{-1}$ ), UVOT must have observed within the first $400 \mathrm{~s}$ until at least $10^{5} \mathrm{~s}$ after the BAT trigger and the colour of the afterglows must not evolve significantly with time, meaning that at no stage should the light curve from a single filter significantly deviate from any other filter light curve when normalized to the $v$ filter. These criteria ensure that a high signal-to-noise (SN) light curve, covering both early and late time evolution, could be constructed from the UVOT multi-filter observations using the method given by [2]. The main steps of this method were to normalize the multi-filter light curves to the $v$ filter and then to group them together using a binsize of $\Delta t / t=0.2$. The requirement for no strong colour evolution 


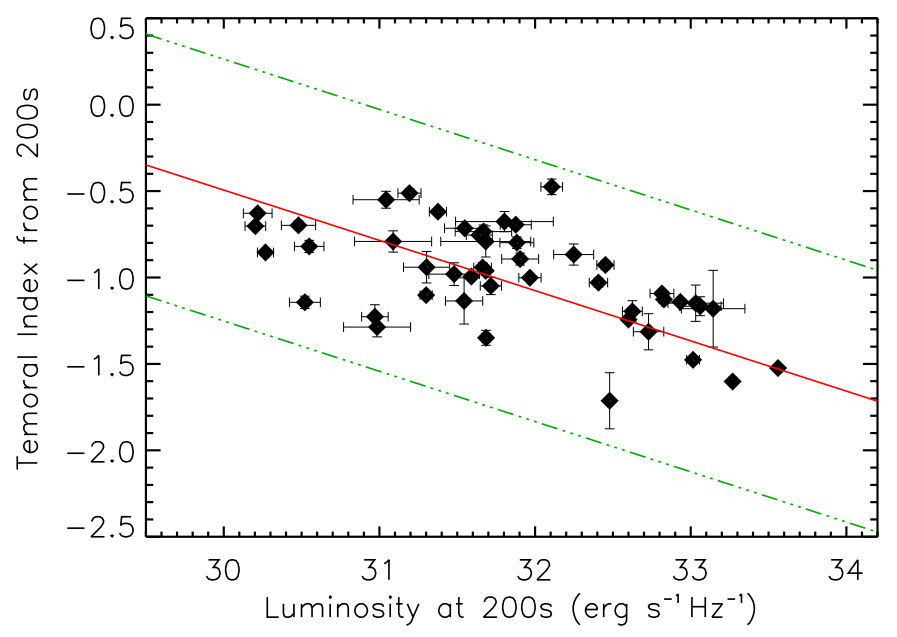

Figure 2: Luminosity at 200s versus average decay determined from the luminosity light curves after 200s. The red solid line shows the best fit regression and the green dotted-dashed line represents the $3 \sigma$ deviation.

ensures that when the multi-filters are normalized to the $v$ filter, the data from the different filters can then be treated as being equivalent to the $v$ band and co-added. Two GRBs in particular fit the selection criteria, but were excluded as they were considered to have strong colour evolution: GRB060218, GRB100814A.

Luminosity light curves were produced for $56 \mathrm{GRBs}$ that had spectroscopic or photometric redshifts and for which the host $\mathrm{E}(\mathrm{B}-\mathrm{V})$ values could be determined. Of the sample of 69 light curves, 15 did not have spectroscopic or photometric redshifts available in the literature. For 2 of these GRBs we were able to derive photometric redshifts from joint XRT-UVOT Spectral Energy Distributions (SEDs), following the method of [П]: $z=1.2 \pm 0.1$ for GRB 060510A and $z=3.1 \pm$ 0.1 for GRB 090401B. The single filter count rate light curves were converted to luminosity at a common restframe wavelength of $1600 \AA$, and were corrected for both Galactic and host extinction. The host extinction was calculated from the host $A_{v}$ values reported in [5]. For those not reported in [5], we used the same method to determine the host $A_{v}$.

\section{Results}

The luminosity light curves at $1600 \AA$, in units of $\mathrm{erg} \mathrm{s}^{-1} \mathrm{~Hz}^{-1}$, are given in Figure 1. The light curves are clustered in a single group. They appear to have the largest range in luminosity at the earliest epochs, which becomes narrower as the light curves decay. This suggests that the most luminous GRBs decay the quickest, while the less luminous GRBs decay more slowly. In order to test for a correlation between intrinsic brightness and decay rate, we determined the logarithm of the brightness at 200s, $\log \mathrm{L}_{200 \mathrm{~s}}$, by fitting a spline function to the data between 100 and $2000 \mathrm{~s}$ and fit a single power law to the light curves from 200s onwards in order to determine the average decay rate, $\alpha_{>200 \mathrm{~s}}$. For this analysis, we wish only to determine the average rate of decay, we do not remove flares or try to account for breaks in the light curve, both of which may add dispersion 
into the correlation. We exclude a further 9 GRBs for which the errors on the decay index were greater than 3 times the mean decay error for the sample as a whole or for which there were too few data points between 100 and 2000s to constrain the luminosity at 200s. The resulting values for $\log \mathrm{L}_{200 \mathrm{~s}}$ and $\alpha_{>200 \mathrm{~s}}$ are shown in Fig. 国. A Spearman Rank test of $\log \mathrm{L}_{200 \mathrm{~s}}$ against $\alpha_{>200 \mathrm{~s}}$, gives a correlation coefficient of -0.54 and a probability of correlation of $99.99 \%(3.9 \sigma)$. This indicates that the two parameters are statistically correlated and confirming our observation that the more luminous optical/UV afterglows decay more quickly than the lower luminosity ones.

We examined if the correlation was a result of the selection criteria by performing a Monte Carlo simulation using $5 \times 10^{5}$ trials. For each trial, we simulated a random distribution of 47 pairs of $\log \mathrm{L}_{200 \mathrm{~s}}$ and $\alpha_{>200 \mathrm{~s}}$. For each pair, we calculated the observed frame light curve, using the values of $\log \mathrm{L}_{200 \mathrm{~s}}$ and $\alpha_{>200 \mathrm{~s}}$, and randomized parameters for redshift, extinction and k-correction. If the resulting observed frame light curve did not meet our selection criteria we discarded the $\log \mathrm{L}_{200 \mathrm{~s}}-\alpha_{>200 \mathrm{~s}}$ data point from the simulated distribution and drew a new pair of values until the selection criteria was met. Once the 47 valid pairs of $\log \mathrm{L}_{200 \mathrm{~s}}$ and $\alpha_{>200 \mathrm{~s}}$ values had been verified, we ran a Spearman Rank correlation on the remaining distribution. We recorded the correlation coefficient and repeated this for each of the $5 \times 10^{5}$ trials.

There are only 18 trials that have a correlation coefficient, equal to or indicating a stronger correlation than that found for the original $\log \mathrm{L}_{200 \mathrm{~s}}-\alpha_{>200 \mathrm{~s}}$ correlation. This indicates that the correlation is not due to our choice of selection criteria at $4 \sigma$ confidence and therefore implies that the $\log \mathrm{L}_{200 \mathrm{~s}}-\alpha_{>200 \mathrm{~s}}$ correlation is an intrinsic property of long GRBs.

\section{Discussion}

Using 47 optical/UV light curves, we have found a significant correlation between optical afterglow luminosity and average decay rate and determined that is an intrinsic property of long GRBs. This correlation appears to be universal for all optical/UV light curves in our sample. We shall now examine three ways to produce a correlation between $\log \mathrm{L}_{200 \mathrm{~s}}$ and $\alpha_{>200 \mathrm{~s}}$.

\subsection{Basic GRB Afterglow Model}

A correlation between $\log \mathrm{L}_{200 \mathrm{~s}}$ and $\alpha_{>200 \mathrm{~s}}$ may be a natural result of the jet interacting with the external medium, which produces synchrotron emission, and which relates the luminosity to $\alpha$ by $L \propto t^{\alpha} v^{\beta}$, where $\beta$ is the spectral index. In this model, the temporal and spectral indices are related linearly to each other by a set of closure relations (e.g [8]). If the $\log \mathrm{L}_{200 \mathrm{~s}}-\alpha_{>200 \mathrm{~s}}$ correlation is simply a result of this afterglow model, then we must examine two scenarios to determine if this is the case. In the most basic scenario, the optical light curves, for all GRBs, are produced from the same spectral regime, implying that the optical afterglows are produced from the same spectral segment and have the same density profile. If this were the case then a single closure relation would describe the relationship between $\alpha$ and $\beta$ and we should expect to see a linear correlation between these two parameters and a correlation between $\log \mathrm{L}_{200 \text { s }}$ and $\beta$. However, we can exclude this scenario as we do not observe either of these correlations in Fig. 3. Moreover, it is unlikely that all optical afterglows are produced by the same spectral regime. In the second scenario, we consider the optical afterglows to be produced by multiple spectral regimes. In Fig. 3 we display the relationship between $\alpha$ and $\beta$, given by the closure relations, for three most likely 


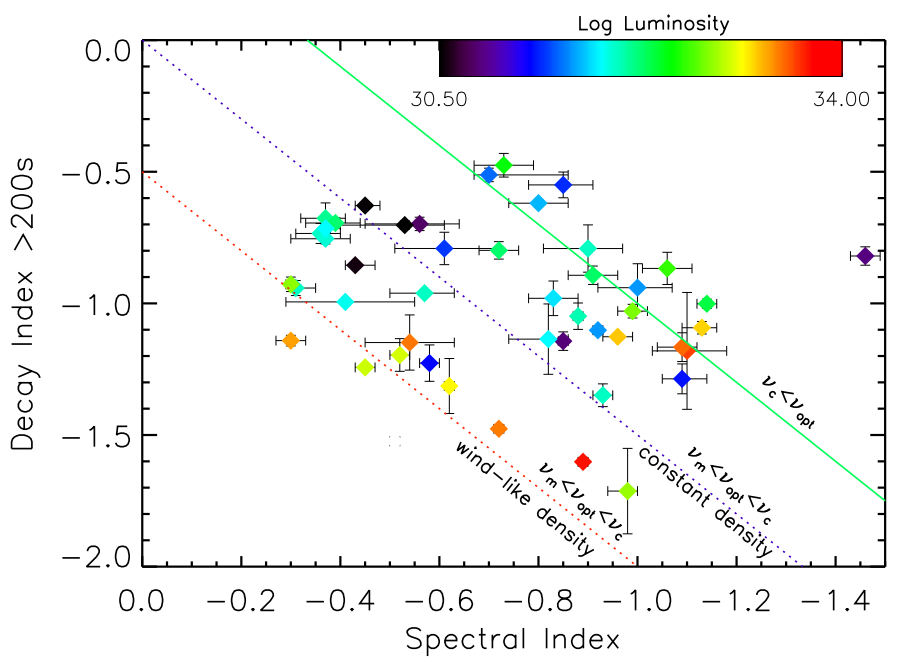

Figure 3: Optical/UV temporal and spectral indices for the 47 GRBs. The lines represent the 3 closure relations that most likely describe the optical afterglows. A colour scale represents the range in $\log \mathrm{L}_{200 \mathrm{~s}}$. No relationship is observed between luminosity and the spectral index, nor temporal and spectral index.

spectral regimes. In this more complex scenario, if the $\log \mathrm{L}_{200 \mathrm{~s}}-\alpha_{>200 \mathrm{~s}}$ relation is a natural result of the standard afterglow model, we should expect to see clustering of the luminosities around a given closure relation, but again we do not observe this in Fig. 3. We thus exclude the standard afterglow model as the cause of the correlation.

\subsection{Complex GRB Afterglow Model}

The standard model is likely to be more complex than we assumed in the previous section and there may be some mechanism or parameter that regulates the energy release in GRB afterglows, depending on the rate of decay. This would be controlled in such a way that if the energy is released quickly the result is a bright-fast decaying afterglow, while if the energy is released more slowly a fainter-slower decaying afterglow is observed. One possible way to regulate this could be continued energy injection. For instance, if the central engine does not release its energy all in one go, but releases it over a much longer duration, then we may expect faint - slowly decaying afterglow.

\subsection{Off-axis and Structured Outflows}

Alternatively, the $\mathrm{L}_{200 \mathrm{~s}}-\alpha_{>200 \mathrm{~s}}$ correlation may instead be a geometric effect resulting from a range in viewing angle, $\theta_{\text {obs }}$. In this scenario, the faint-slowly decaying optical afterglows would be those observed at the largest angles, while the bright-fast decaying light curves would be observed within the outflow (e.g [7, 61). [6] show, in their Fig. 3., that, for a jet with uniform velocity distribution, an off-axis observer, i.e $\theta_{\text {obs }} / \theta_{\text {jet }}>1$, where $\theta_{\text {jet }}$ is the jet opening angle, will observe a shallower decay and observe the afterglow to be less luminous in comparison to an observer who is observing closer to the edge of the jet, i.e $\theta_{\text {obs }} / \theta_{\text {jet }} \sim 1$. This effect should be observed for both constant density and wind-like external media. This model is more complex if structured outflows 
are considered. In this scenario, the off-axis viewers will observe a shallower and fainter light curve in comparison with on-axis observers, just like for the isotropic jet, but the convergence time and the range of light curve decays will vary, depending on how the energy is distributed within the jet.

\section{Conclusions}

We have computed luminosity light curves at $1600 \AA$ for a sample of 47 optical/UV GRB afterglows and find a correlation between luminosity at 200s and average decay rate determined from 200s onwards with a significance of $99.99 \%$ (3.9 $\sigma$ ). We determine using a Monte Carlo simulation that this is an intrinsic property of the optical afterglows of GRBs. We give two possible causes. The first may be that there is an intrinsic mechanism or physical quantity that controls how quickly the energy is released, such that bright, fast-decaying afterglows release their energy more quickly than faint, slow-decaying afterglows. Alternatively, the energy is released at a similar rate in all afterglows, but we are observing the jets over a wide range of angles so the fainter, slowerdecaying afterglows are viewed at larger angles than the brighter, faster-decaying afterglows.

Understanding the origin of this correlation will have important consequences on our understanding of the physics and geometry behind these GRBs. In addition, upon further investigation and inclusion of a larger sample, we may be able to use this correlation to derive independent constraints on the redshift of GRBs using only the decay rate of the optical afterglow.

\section{Acknowledgments}

This research has made use of data from the High Energy Astrophysics Science Archive Research Center (HEASARC) and the Leicester Database and Archive Service (LEDAS), provided by NASA's Goddard Space Flight Center and the Department of Physics and Astronomy, Leicester University, UK, respectively. SRO and MJP acknowledge the support of the UK Space Agency.

\section{References}

[1] Gehrels. N, et al., The Swift Gamma-Ray Burst Mission, ApJ 2004 (611) 1005

[2] Oates. S. R, et al., A statistical study of gamma-ray burst afterglows measured by the Swift Ultraviolet Optical Telescope, MNRAS 2009 (395) 490 [ast ro-ph/ 0901.3597 ]

[3] Roming et al., The second UVOT Gamma-ray Burst Catalog, in preparation, 2005

[4] Krühler T. et al., The SEDs and host galaxies of the dustiest GRB afterglows, A\&A 2011 (526) A153

[5] Schady P. et al., Dust and metal column densities in gamma-ray burst host galaxies, MNRAS 2010 (401) 2773 [astro-ph/0910.2590]

[6] Panaitescu, A. \& Vestrand, W. T., Taxonomy of gamma-ray burst optical light curves: identification of a salient class of early afterglows, MNRAS 2008 (387) 497 [astro-ph / 0803.1872 ]

[7] Ramirez-Ruiz, E., An Off-Axis Model of GRB 031203, ApJL 2005 (625) L91

[astro-ph/0412145]

[8] Zhang. B., et al., Physical Processes Shaping Gamma-Ray Burst X-Ray Afterglow Light Curves: Theoretical Implications from the Swift X-Ray Telescope Observations, ApJ 2006 (642) 354 [astro-ph/0508321] 